\section{Combining Focused Ion Beam and Scanning Electron Microscopy for IC Fab Support and Defect Review}

Janet Tashima \& Dr. Jay Lindquist, FEI Company

The cutting-edge tool for $\mathrm{IC}$ fab support and defect review brings together the Focused lon Beam $(F \mid B)$ technology with the Scanning Electron Microscope (SEM) into a single workstation. The twin beam FIB/SEM workstation, FEl Company's DualBeam ${ }^{T M} 820$ for example, combines the unique micromachining, microdeposition, and analysis capabilities of a FIB with the high resolution imaging power of a Schottky field emission scanning electron microscope (FE SEM). The full functionality of both instruments is available and neither is compromised by the other

The combination of these two indispensable fab support tools is enhanced by an easy-to-use operating system and a high-accuracy stage that supports $200 \mathrm{~mm}$ wafers. Both the FIB and SEM are full-featured, and neither technique is compromised by the other. The applications include:

- FIB micromachining (enhanced with selective gas chemistries) for cross-sectioning, transmission electron microscopy (TEM) sample preparation, and defect characterization.

- FIB microdeposition of both metals and dielectrics for IC design modification and testing.

- High-resolution, low-voltage Schottky FE SEM for defect review, CD measurement, and imaging of sub-micron particles and features.

- Energy dispersive x-ray spectroscopy (EDS) for elemental analysis of defects, particles, and features of interest.

\section{The DualBeam's Role in Defect Review}

Using defect review tools (DRTs) connected to wafer inspection systems, the yield management engineer can classify defects by size, shape, or type. Defect data and yield management software systems coordinate the massive amount of data that results from testing and inspection. Using a data and yield management system, engineers can determine which defects affect yield and choose examples of "killer" defects for further characteriza-

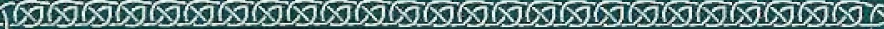 APPLICATIONS/DEMONSTRATION SPECIALIST}

Nissei Sangyo America, Ltd./Hitachi Scientific Instruments, an EEOE, is seeking a skilled and motivated individual to join our Washington, D.C. Demo Lab.

The successful candidate will be responsible for performing SEM demonstrations, and providing technical support for our customers and sales force. Based in Gaithersburg, MD, the position requires the ability to travel up to 60 percent of the time.

Excellent verbal and written communication skills, and broad-based experience in scanning electron microscopy are required. Experience with variable pressure and field emission scanning electron microscopes is a plus.

For confidential consideration, send professional resume including salary requirements to:

APPLICATIONS SPECIALIST

Nissei Sangyo America/Hitachi 25 West Watkins Mill Road, Suite $\mathrm{H}$ Gaithersburg, MD 20878 tion.

In a typical defect review sequence, an optical wafer inspection system (Tencor, KLA, etc.) scans a wafer for defects and records their locations. An operator, using an SEM with semi-automated classification tools, classifies the defects - and may tag the original defect file, attaching an image of the defect and verifying its size and location. Engineers then check for correlations and process yield enhancements. If a systematic problem arises, wafers are pulled for defect characterization, cross-sectioned in a FIB Workstation, then analyzed in an SEM. The defect's composition is determined, typically by EDS, and this information is attached to the original defect file. Process, test, and yield management engineers use the characterization data to determine the defect's source.

The SEM capabilities of the DualBeam 820 FIB/SEM Workstation are optimized for two-dimensional defect review and classification (2D-DR). Defect data files (The DualBeam software accepts data from all major inspection tools.) are transferred via the network connection, and the stage navigates with submicron accuracy to the defect coordinates. The vertically mounted electron column provides high-resolution images or low $\mathrm{kV}$ - and EDS spectra, if warranted. (The DualBeam interfaces with all major EDS vendors.) This information can be tagged back to the original defect file.

The

FIB capabilities of DualBeam make possible an advanced technique - threedimensional defect review and characterization (3D-DR). Using the same navigation techniques as in the $2 \mathrm{D}$-DR mode, the FIB can rapidly uncover or cross-section defects for FIB, SEM, and EDS imaging and analysis. This information can be tagged back to the original defect file

The DualBeam's multi-tiered user interface offers simple operation for routine tasks coupled with secured access for sensitive functions and usage tacking by senior engineering, maintenance, and management staff members. There is an optional cleanroom configuration offering cassette wafer handling, low particles per wafer pass (PPWP) contamination contribution, a chasecompatible reduced-area footprint, cleanroom final assembly, and double-wrap packaging.

\section{Unique Capabilities and Practical Advantages of the DualBeam}

The DualBeam 820 offers several unique advantages because of the synergy of the FIB, FE SEM, $200 \mathrm{~mm}$ stage, operating software, and EDS features. Higher throughput is achieved with the DualBeam because it:

- Eliminates transfer time between different systems and the repeated navigation to specific feature locations.

- Allows for simultaneous FIB milling and SEM viewing of cross section.

- Provides an efficient classification process - overlaying defect locations onto the die CAD map - to reveal "killer" defects.

- Streamlines defect characterization using the complementary information gained from SEM, FIB, and EDS imaging and analysis

- Puts FIB sample preparation and SEM or EDS characterization in the same system.

The DualBeam's advantages also include a reduced cost of ownership compared to independent systems with matching features. This is primarily due to the Workstation's lower purchase price and smaller footprint versus multiple systems. Other factors include reduced operator training time as FIB and SEM controls are completely integrated and an inherent reduction in PPWP using the DualBeam.

$\star \star \star \star \star \star ⿱ 亠 乂$
$\star$



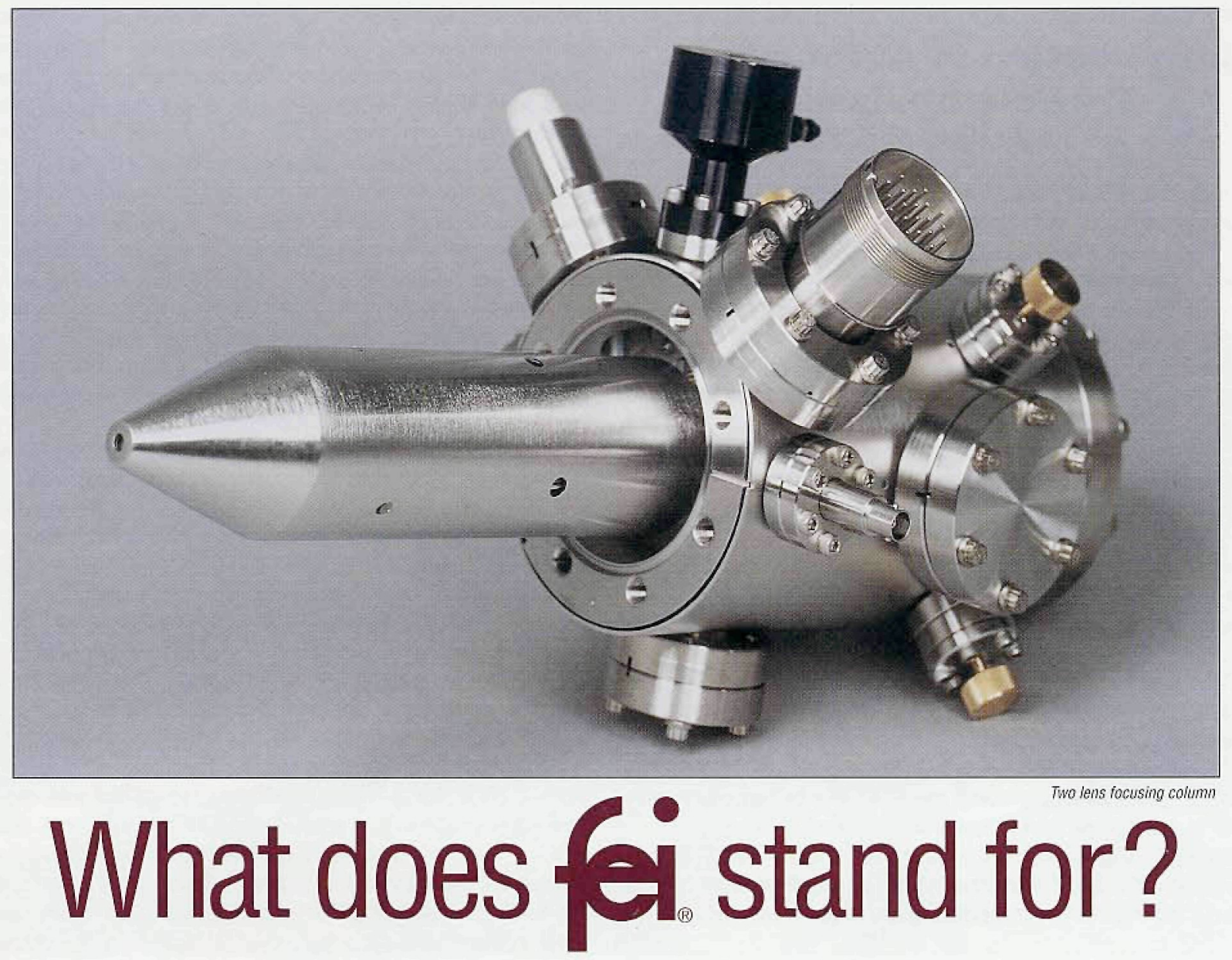

Our compact, UHV, field emission columns are used by researchers world wide. Innovative electrostatic optics and dedicated electronics allow you to integrate a high current density electron or ion column into most vacuum systems. FEI also supplies researchers with other specialized products...

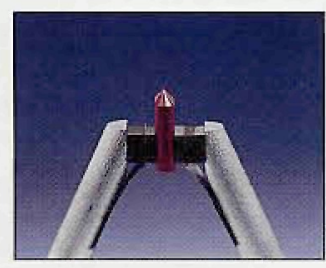

\section{$\mathrm{LaB}_{6}$ an $\mathrm{CeB}_{6}$ Cathodes}

FEl's Mini-Vogel Mount, the first universally compatible long-life, high-stability LaB6 cathode, provides excellent performance and the best cost-per-use value for installation into your EM systems.

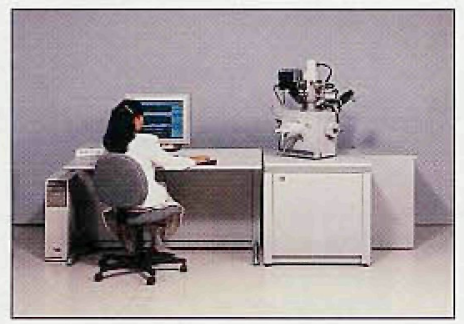

\section{FIB Workstations}

Focused ion beam micromilling workstations range from the 8"-wafer compatible model to the economical 2" small sample model ideal for semiconductor, biological, TEM, and MEMS specimens.

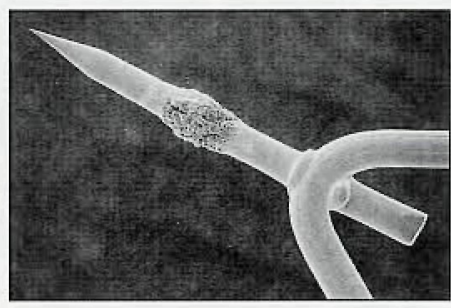

Field Emission Cathodes

FEl supplies Schottky field emitters to EM manufacturers worldwide. Schottky emission's high current intensity has established it as the preferred electron source for high resolution SEM, TEM, Auger, ESCA, EDX, and lithography.

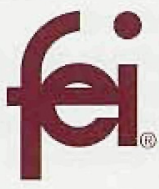

FEI Company

7451 NE Evergreen Parkway

Hillsboro, OR 97124-5830

(503) 640-7500 Fax (503) 640-7509

email:rsk@feico.com

Now, when you think of FEl, you'll know we are the Specialists in Field Electron and Ion Technology. 